\title{
Réunion Island prepared for possible Zika virus emergence, 2016
}

S Larrieu ${ }^{1}$, L Filleul ${ }^{1}$, $O$ Reilhes ${ }^{2}$, M Jaffar-Bandjee ${ }^{3}$, C Dumont ${ }^{4}$, T Abossolo ${ }^{4}$, H Thebault ${ }^{2}$, E Brottet ${ }^{1}$, F Pagès ${ }^{1}$, P Vilain ${ }^{1}$, I Leparc-Goffart ${ }^{5}$, E Antok ${ }^{6}$, D Vandroux ${ }^{6}$, P Poubeau ${ }^{7}, M_{\text {Moiton }}^{7}$, P Von Theobald ${ }^{4}$, F Chieze ${ }^{2}$, A Gallay $^{8}$, H De Valk $^{8}$, F Bourdillon 8

1. Santé publique France, French national public health agency, Regional unit (Cire) Océan Indien, Réunion, France

2. Agence de Santé Océan Indien, Saint Denis, Réunion, France

3. CHU de la Réunion, Centre National de Référence des Arbovirus, Réunion, France

4. CHU de la Réunion, Centre de Diagnostic Prénatal, Réunion, France

5. Centre National de Référence des Arbovirus, Irba, Marseille, France

6. CHU de la Réunion, Service de Réanimation, Réunion, France

7. CHU de la Réunion, Service de Maladies infectieuses, Réunion, France

8. Santé publique France, French national public health agency, Saint-Maurice, France

Correspondence: Sophie Larrieu (sophie.larrieu@ars.sante.fr)

Citation style for this article:

Larrieu S, Filleul L, Reilhes O, Jaffar-Bandjee M, Dumont C, Abossolo T, Thebault H, Brottet E, Pagès F, Vilain P, Leparc-Goffart I, Antok E, Vandroux D, Poubeau

P, Moiton M, Von Theobald P, Chieze F, Gallay A, De Valk H, Bourdillon F. Réunion Island prepared for possible Zika virus emergence, 2016 . Euro Surveill.

2016;21(28):pii=30281. DOI: http://dx.doi.org/10.2807/1560-7917.ES.2016.21.28.30281

Article submitted on 15 March 2016 / accepted on 05 July 2016 / published on 14 July 2016

Zika virus (ZIKV) has recently spread widely and turned into a major international public health threat. Réunion appears to offer conditions particularly favourable to its emergence and therefore prepared to face possible introduction of the virus. We designed a scaled surveillance and response system with specific objectives, methods and measures for various epidemiological phases including a potential epidemic. Several tools were developed in order to (i) detect individual cases (including a large information campaign on the disease and suspicion criteria), (ii) monitor an outbreak through several complementary systems allowing to monitor trends in disease occurrence and geographic spread and (iii) detect severe forms of the disease in collaboration with hospital clinicians. We put the emphasis on detecting the first cases in order to contain the spread of the virus as much as possible and try to avoid progress towards an epidemic. Our two main strengths are a powerful vector control team, and a close collaboration between clinicians, virologists, epidemiologists, entomologists and public health authorities. Our planned surveillance system could be relevant to Europe and island settings threatened by Zika virus all over the world.

\section{Introduction}

Zika virus (ZIKV) is a mosquito-borne flavivirus transmitted by Aedes spp. mosquitoes. It was first isolated in 1947 from a sentinel Rhesus monkey in Uganda [1], and shortly hereafter it was shown to cause human infections $[2,3]$. During sixty years, its area of distribution has been restricted to Africa and Asia where it has been recognised to be a cause of febrile illness in humans with symptoms including fever, headache, conjunctivitis, myalgia, rash, joint pains [4,5]. In 2007, it spread outside its usual geographic range for the first time, and caused an outbreak on Yap Island in the Federated States of Micronesia [4]. Most infected persons were asymptomatic or had a mild disease. Therefore, despite an attack rate of $73 \%$, international authorities and the media paid little attention to this event.

However, this emergence of ZIKV outside Africa and Asia was a warning signal of its potential to spread to other Pacific islands and the Americas [5]. In 2013-14, the virus continued its geographical expansion and caused large outbreaks in the western Pacific region, including French Polynesia, New Caledonia, Easter Island, and the Cook Islands [6-9]. International concern was raised when the virus was suspected to be associated with a 20 -fold increase of Guillain-Barré syndrome (GBS) incidence in French Polynesia [10]. In 2015, ZIKV reached the Americas. After ZIKV emerged in Brazil, an increase in suspected microcephaly cases and other fetal anomalies was observed and thought to be associated with ZIKV infection during pregnancy. This caused Brazil to declare Zika a public health emergency of national importance in November 2015 [11].

In just three years, a virus considered benign turned into a major public health threat because of its impressive capacity to spread out rapidly, the associated high attack rates and its ability to cause severe illness. In that context, the World Health Organization (WHO) encouraged countries at risk to be prepared for ZIKV emergence in terms of diagnosis, surveillance and vector control [12]. On 1 February 2016, following a meeting of its 'International Health Regulations (2005) 
Emergency Committee on Zika virus and observed increase in neurological disorders and neonatal malformations', WHO declared that the ZIKV epidemic, constitutes a Public Health Emergency of International Concern (PHEIC) [13].

Réunion, a French overseas administrated territory with 830,000 inhabitants located in the south-western Indian Ocean, faced a similar arbovirus threat in 200607. A hardly known arbovirus called chikungunya virus, considered as benign at the time, emerged on the island and caused the largest epidemic ever described with an attack rate of $34 \%$ [14] and occurrence of unexpected severe forms, which led to a major health and social crisis. Ten years later, Réunion appears to offer conditions particularly favourable to emergence of ZIKV. Indeed, ZIKV circulation has never been documented in Réunion and therefore immunity may be very low, or even non-existent in the general population. The main vector for Zika virus is Aedes agypti, however, Ae. albopictus, abundant throughout the year in all inhabited areas of the island was also described as a competent vector of ZIKV [15,16]. Therefore, Réunion prepared for possible ZIKV emergence by implementing a surveillance system in order to limit the risk of spread on the whole island.

The aim of this paper is to describe the tools set up in Réunion in order to face a potential emergence of ZIKV within the next few months.

\section{Overview of the surveillance and response system}

Réunion benefits from a healthcare system similar to mainland France with more than 890 general practitioners and 80 paediatricians distributed throughout the island, 59 laboratories, three public and 56 private, as well as four hospitals and six emergency departments.

In November 2015, a specific surveillance system of ZIKV infections was implemented on Réunion by the regional unit (Cire OI) of the French national public health agency (Santé publique France) in collaboration with the French Health Agency Indian Ocean (ARS OI). It is based on current knowledge about ZIKV and past experience with chikungunya and dengue surveillance and control and follows the WHO recommendation [12]. We designed a scaled surveillance and response system with specific objectives, methods and measures for various epidemiological phases including a potential epidemic (Figure 1).

The focus is on three surveillance objectives:

(i) To detect individual cases at any time, except during an outbreak;

(ii) To monitor the outbreak i.e. to follow trends in disease occurrence and document the health impact;

(iii) To detect and describe severe forms of the disease.
Detection of individual cases

The general organisation of the surveillance system implemented to detect all ZIKV cases is presented in Figure 2. Surveillance is based on the notification by the reference laboratory and health practitioners of any suspected and confirmed cases of ZIKV.

A patient has to meet both clinical and epidemiological criteria to be considered as a suspected case:

(i) Clinical criteria: patient presenting with maculopapular rash with or without fever AND at least two additional symptoms (conjunctivitis/arthralgia/myalgia);

(ii) Epidemiological criteria: travel in an area affected by ZIKV circulation within the two weeks preceding clinical symptoms; any temporo-spatial cluster of patients meeting the clinical criteria.

A probable case is a suspected case with presence of IgM antibody against Zika virus and an epidemiological link with any confirmed case.

A confirmed case is a person with laboratory confirmation of recent Zika virus infection i.e. positive reverse transcriptase (RT)-PCR in serum or other sample or positive seroneutralisation assay and exclusion of other flaviviruses.

The Regional and National Reference Laboratories for Arboviruses perform the laboratory diagnosis of Zika virus infection. It is based on the direct detection of viral genome by RT-PCR on serum from day o to 5 after symptom onset and on urine until day 15 after symptom onset. The detection of IgM and IgG anti-Zika on serum from day 5 after the onset of symptoms is complicated by the frequency of cross-reactions with other flaviviruses such as dengue virus. The detection of antibodies could be confirmed by seroneutralisation assay to determine the specificity of the detected antibodies. Suspected cases should also be tested for dengue and chikungunya as both these arboviruses could emerge or re-emerge in Réunion.

As soon as a case is notified, after a brief assessment by the Cire OI, control measures are immediately implemented by the vector control team. They comprise the elimination of peri-domiciliary breeding sites of Aedes mosquitoes, spraying, health education, and active door-to-door case finding. Considering expected $A e$. albopictus area of activity, such control measures are performed in a $100 \mathrm{~m}$ radius around the residence of the case (i.e. the expected radius of activity of the mosquito) and around any subsequent suspect cases identified during the case finding. They can also be implemented around the worksite, or any place where cases declared having spent time or been exposed to mosquitoes. An entomologic surveillance using house and Breteau indexes [17] is routinely performed through control of a random sample of almost 50,000 houses per year i.e. $15 \%$ of the total dwellings of the island. 


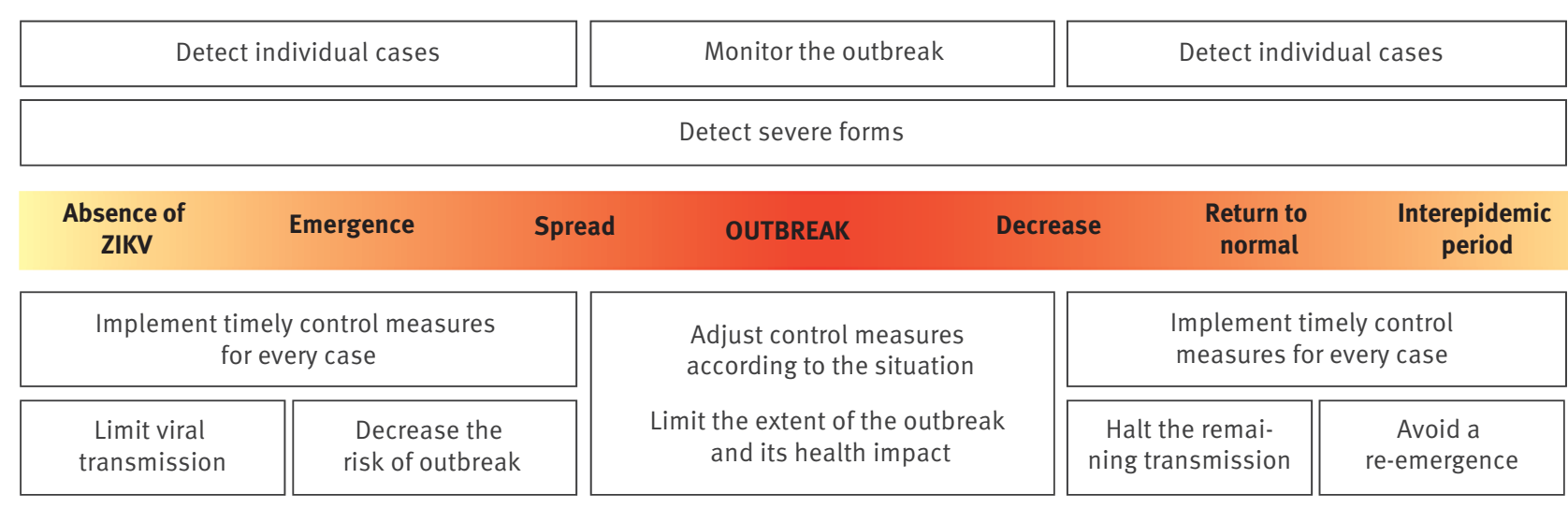

\section{RESPONSE OBJECTIVES}

ZIKV: Zika virus.

In parallel, exercises are performed in order to check effectiveness of control measures on vector densities.

As this surveillance is based on detection of suspect cases by general practitioners (GPs), we undertook an information campaign in order to raise their awareness of ZIKV and its potential emergence. A document was delivered in person to all the GPs on the island, focusing on the disease (clinical signs, transmission and laboratory diagnosis) and including an epidemiological report presenting the international situation, the risk for Réunion and recommendations on case detection. Updated versions of this document are sent by email and can be uploaded from the ARS webpage. We also organised numerous specific meetings with the medical staff of intensive care units as well as emergency, gynaecology, and infectious diseases departments.

Furthermore, this information was relayed by the media in order to inform the general population, and especially travellers returning from an epidemic area. A poster was displayed at the airport, presenting symptoms associated with ZIKV infection and recommending people returning from an affected area to visit a doctor in case of any of these symptoms.

In case of laboratory confirmation of an autochtonous case, all GPs of the affected area(s) are immediately called by phone in order to enhance their vigilance and promptness of case notification.

When autochthonous transmission of ZIKV is identified on the island, the objective of surveillance remains the same i.e. to detect all the cases in order to implement individual control measures. The organisation of the surveillance systems and the control measures remain unchanged. However, in this instance, all inhabitants of Réunion meet the epidemiological criteria of the case definition and suspicion is based on clinical criteria only.

\section{Monitoring the outbreak}

During the outbreak phase, laboratory confirmation of each individual case and implementation of control measures around each case are no longer efficient nor feasible. Therefore, surveillance shifts to a sentinel monitoring of trends in disease occurrence and the geographic spread, based on several complementary surveillance systems: (i) a network of sentinel general practitioners, (ii) online self-reported symptoms surveillance, (iii) concern about Zika on social media, (iv) syndromic surveillance of emergency department consultations, (v) mortality surveillance. Control measures are guided by epidemiological surveillance, with more vector control teams in the most affected areas in order to limit local transmission of the virus.

\section{Sentinel practitioners network}

On Réunion, a sentinel network, consisting of $50 \mathrm{GPs}$ scattered across the island and representing $6.2 \%$ of the GPs on Réunion, is operational since 1996 and conducts surveillance of influenza, gastroenteritis and varicella [18]. On a weekly basis, they report the number of consultations for these syndromes.

This network can be rapidly mobilised to monitor other diseases with an epidemic potential, and has already shown its responsiveness and reliability for surveillance during outbreaks of chikungunya in 2005-06 [14], pandemic influenza $A\left(\mathrm{H}_{1} \mathrm{~N}_{1}\right)$ in 2009 [19], gastroenteritis in 2012 [20] and conjunctivitis in 2015 [21]. This network will be activated for the surveillance of suspected cases of ZIKV infections in case of an 


\section{FIGURE 2}

General organisation of the surveillance system to detect individual ZIKV cases, Réunion, 2016

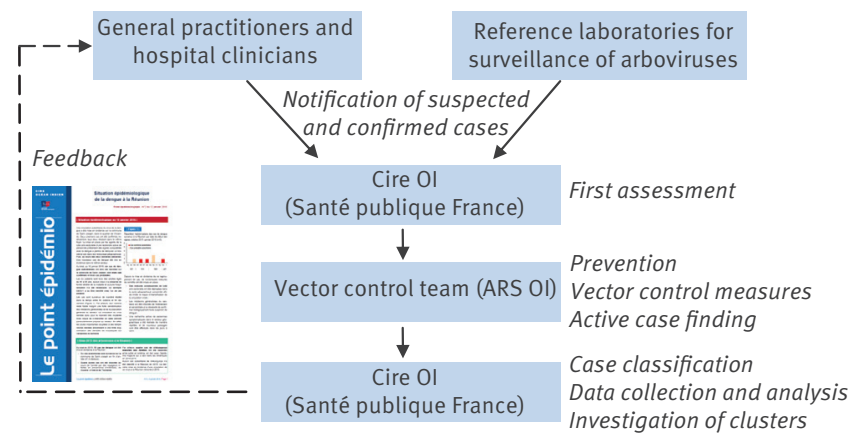

ARS OI: French Health Agency Indian Ocean; Cire OI: Regional unit of the French national public health agency (Santé publique France)

outbreak in Réunion, and the total number of symptomatic ZIKV cases can be estimated on a weekly basis.

Online self-reported symptoms surveillance

The ARS OI implemented in April 2014 a web-based surveillance system called 'Koman i lé', that allows to follow perceived health among people who do not systematically visit their GP. Individual volunteers aged over 18 weekly fill in a short survey asking symptoms presented during the previous week. An indicator for ZIKV infection has been constructed based on clinical criteria previously presented: rash with or without fever AND at least two additional symptoms (conjunctivitis/arthralgia/myalgia). The total number and proportion of participants reporting ZIKV symptoms can be monitored on a weekly basis, for the whole island and according to place of residence.

Concern about Zika virus on social media

Several studies suggest that social media could be useful to monitor epidemics of infectious diseases [22]. Sick people notably talk on Twitter about their health conditions, their feelings about the symptoms, and treatments they take to relieve the symptoms. In that context, a tool was developed to monitor tweets concerning Zika posted by Réunion dwellers. Tweets mentioning the keyword 'Zika' are collected using an $\mathrm{R}$ programme from the free Twitter public application programming interface (API), which allows individuals to request a feed of public tweets matching specific search criteria. Then, usernames are removed in order to anonymise the tweets. Duplicate tweets and retweets (tweets posted by one user and then forwarded by another user) are removed. Then, the weekly number of tweets concerning Zika on the island can be obtained and monitored such as other surveillance indicators. Text mining can also be performed in order to explore qualitatively the concern of the population. The goal of this surveillance is to measure the concern of the population about Zika virus, which could be associated with the real incidence of the disease.
Emergency department consultations

Réunion has a syndromic surveillance system based on all four emergency departments (ED) of the island (Organisation de la surveillance coordonnée des urgences (OSCOUR) network). Data are collected each day directly from the patients' computerised medical files that are filled in during medical consultations at the ED. Each morning, data are downloaded and analysed by an epidemiologist of the Cire OI. The diagnoses are classified according to the 1oth revision of the International Classification of Diseases (ICD-10) [23]. The risk of emergence of Zika virus was recently discussed with the staff of the four EDs of the island. In case of emergence, specific meetings will immediately be organised in order to increase awareness, and remind healthcare workers about the case definition and ICD-10 classification code for suspicion of ZIKV.

Zika virus-associated and all-cause mortality

In case of an outbreak, the total number and excess of deaths from all causes will be analysed on a weekly basis in order to detect a potential increase. This system will be completed by analysis of all death certificates received by the regional public health authority that mention 'Zika'.

\section{Surveillance of severe cases}

Based on current knowledge on severe forms potentially associated with ZIKV infection [10,24], two specific systems were implemented: (i) surveillance of GBS and other neurological disorders and (ii) surveillance of microcephaly cases and other fetal anomalies, to monitor the number of cases, to describe the patients and their evolution, and to detect any emerging severe form of the disease. The observed number of cases will be compared with the expected number to determine whether a significant increase is observed.

All suspicions of severe cases will lead to laboratory investigations of serum and urine in order to document ZIKV infection by RT-PCR, detection of antibodies and seroneutralisation assay. RT-PCR will also be performed on cerebrospinal fluid for GBS and other neurological diseases, and on amniotic fluid and fetal tissues (in case of fetal loss) for fetal anomalies.

\section{Surveillance of Guillain-Barré syndrome and other} neurological forms

A specific surveillance system has been developed with hospital clinicians from departments likely to hospitalise such patients: adult and paediatric intensive care units, infectious diseases and neurology departments. All cases of GBS and other potential neurological complications (meningo-encephalitis, myelitis, etc.) will be notified in real time to the Cire Ol through a specific form. Collected data will include: socio-demographic information, comorbidities, type of neurological disease, symptoms of ZIKV infection during the previous weeks, laboratory results, length of hospitalisation and evolution. The number of expected GBS cases has been estimated using hospital databases. From 2010 
to 2014 , the mean number of yearly reported cases was 17, the median was 20 and the median number of monthly cases ranged from o to 5 without variations between the rainy and the dry season.

Surveillance of microcephaly and other fetal anomalies On Réunion, suspicions of microcephaly or other fetal anomaly detected by ultrasound are systematically referred to one of the two prenatal diagnostic centres of the island. All cases of microcephaly (head circumference under three standard deviations expected for gestation) and/or other brain anomaly potentially linked to ZIKV infection (i.e. in absence of another clearly identified aetiology) will be notified in real time to the Cire OI. Collected data will include: socio-demographic information, comorbidities, type of anomaly, symptoms of ZIKV infection during pregnancy, laboratory results and outcome of pregnancy. Data from the registry of congenital anomalies will also be analysed on a monthly basis in order to detect any increase in microcephaly or other congenital anomalies. The number of expected microcephaly has been estimated using the Eurocat website database [25]. From 2002 to 2012, 56 microcephaly cases were reported on Réunion (average of 8 cases per year, i.e. 3.5 per 10,000 pregnancies). According to those data, the number of expected microcephaly per year is five. As the database showed a non-significant increase of the reported cases in the most recent years, a new estimation was made using data from 2008 to 2012 . According to the most recent reports, the number of expected microcephaly on Réunion is eight per year.

\section{Discussion}

When ZIKV emerged in Brazil, modelling anticipated a significant international spread by travellers to the rest of the Americas, Europe, and Asia [26]. Spread in the Americas is ongoing [27], revealing the explosive pandemic potential of ZIKV. It can be expected that it will emerge soon in other areas of the world and notably in the Indian Ocean. Taking advantage of its unfortunate history of the CHIKV outbreak, Réunion prepared to face a ZIKV emergence within the next months. A surveillance system was implemented to be able to detect the introduction of ZIKV at an early stage and to monitor the spread and impact of the infections in order to guide the implementation of control measures.

Réunion belongs to a regional network for epidemiological surveillance and health alert management coordinated by the Indian Ocean Commission, an intergovernmental organisation including Madagascar, Comoros, Mauritius and Seychelles. All information regarding preparation for ZIKV emergence is shared as part of this network. In case of clinical suspicions in a member country, cooperation can be undertaken for epidemiological investigations and laboratory diagnostics.

\section{Limitations}

Although surveillance of ZIKV has been planned ahead and is based on solid experience and networks [18], some limits can be anticipated. The beginning of the outbreak phase can be demanding for surveillance, particularly when the number of cases cannot be counted anymore but needs to be estimated through GP consultations. Indeed, estimations can lack reliability if the total number of cases remains limited. In such instance, additional GPs will be recruited temporarily in order to improve coverage of the sentinel network, as it was done in 2007 during the chikungunya outbreak. Indeed, some doctors do not want to enrol in the network on long-term basis but volunteer for a temporary participation in case of a specific health event. Also, the suitability of data generated by monitoring Twitter is quite uncertain and will depend on the number of active users of this social network. Work is underway to extend surveillance to other tools more commonly used in Reunion Island, such as Facebook which is particularly popular. Mortality surveillance could lead to over-reporting in case of a large outbreak. The surveillance indicator will be the number of death certificates mentioning the word 'Zika', rather than 'death associated with Zika'; and a clear communication will be essential in order to explain that not all deaths in those infected can be directly attributed to ZIKV infection.

\section{Conclusions}

A large outbreak could have severe effects on the healthcare system and public health infrastructure and would potentially affect general functions of society. Therefore, surveillance and control of ZIKV infections are being anticipated with emphasis on detecting the first cases in order to contain the spread of the virus as much as possible and try to avoid progress towards an epidemic. With this in mind, our two main strengths are:

(i) A powerful vector control team: following the chikungunya outbreak, 150 staff have been employed and trained to fight against arboviruses. They also convey prevention messages and perform active case finding of suspect cases among a large perimeter around every confirmed cases. Considering the subclinical nature of the infection by ZIKV, this active door-to-door case finding is a major asset for early detection of spatiotemporal clusters.

(ii) A close collaboration between clinicians, virologists, epidemiologists, entomologists and public health authorities. Indeed, the 2005-06 chikungunya epidemic led to a major health and social crisis in 2006, and all the local health professionals are conscious of the threat and of the necessity to detect and report the first cases.

Our surveillance system could be relevant to Europe and island settings threatened by Zika virus all over the world. Recently we had the opportunity to test this surveillance system and to show its reactivity and 
effectiveness. Early March, a traveller returned from Martinique on a Saturday morning with fever, rash and arthralgia. About one hour later, they were seen by a clinician who suspected ZIKV infection, initiated laboratory test for confirmation and immediately informed the ARS and the Cire OI. The first control measures around the patient could be undertaken without delay, including confinement and individual protection against mosquito bites. During the following days, peri-domiciliary elimination of breeding sites and spraying was also performed. In April, a second imported case was also detected and confirmed very early. No secondary case was detected or reported despite an active research of symptomatic patients. The surveillance system, and notably information given to health professionals, allowed a timely detection of these cases, and the risk of dissemination was considerably decreased by implementation of immediate control measures.

\section{Acknowledgements}

We would like to thank the CVAGS of the ARS OI, the sentinel doctors, the hospital clinicians of emergency, prenatal diagnosis, intensive care and infectious disease units, and the staff of the Reference Laboratories and the public hospital microbiological laboratory of virology for their high commitment. We are also grateful to all the health professionals participating in the surveillance system: general practitioners, hospital clinicians and private microbiological laboratories.

\section{Conflict of interest}

None declared

\section{Authors' contributions}

SL wrote the manuscript. LF, OR, CD, TA, HT, EB, FP, PV, EA, DV, PP, MPM, PVT, FC, AG, HDV and FB took part in alert and surveillance systems of Zika. MCJB and ILG collaborated in molecular biology techniques. All authors participated in the preparation of Zika surveillance. All authors read and approved the final manuscript.

\section{References}

1. Dick GW, Kitchen SF, Haddow AJ. Zika virus. I. Isolations and serological specificity.Trans R Soc Trop Med Hyg. 1952;46(5):509-20. DOI: 10.1016/0035-9203(52)90042-4 PMID: 12995440

2. MacNamara FN. Zika virus: a report on three cases of human infection during an epidemic of jaundice in Nigeria.Trans $R$ Soc Trop Med Hyg. 1954;48(2):139-45. DOI: 10.1016/00359203(54)90006-1 PMID: 13157159

3. Simpson DI. Zika virus infection in man.Trans R Soc Trop Med Hyg. 1964;58(4):335-8. DOI: 10.1016/0035-9203(64)90201-9 PMID: 14175744

4. Duffy MR, Chen TH, Hancock WT, Powers AM, Kool IL, Lanciotti RS, et al. Zika virus outbreak on Yap Island, Federated States of Micronesia. N Engl J Med. 2009;360(24):2536-43. DOI: 10.1056/NEJMoao805715 PMID: 19516034

5. Hayes EB. Zika virus outside Africa.Emerg Infect Dis. 2009;15(9):1347-50. DOI: 10.3201/eid1509.090442 PMID: 19788800

6. Cao-Lormeau VM, Roche C, Teissier A, Robin E, Berry AL, Mallet HP, et al. Zika virus, French polynesia, South pacific, 2013. Emerg Infect Dis. 2014;20(6):1085-6. DOI: 10.3201/ eid2006.140138 PMID: 24856001
7. Hancock WT, Marfel M, Bel M. Zika virus, French Polynesia, South Pacific, 2013.Emerg Infect Dis. 2014;20(11):1960. DOI: 10.3201/eid2011.141253 PMID: 25341051

8. Ioos S, Mallet HP, Leparc Goffart I, Gauthier V, Cardoso T, Herida M. Current Zika virus epidemiology and recent epidemics. Med Mal Infect. 2014;44(7):302-7. DOI: 10.1016/j. medmal.2014.04.008 PMID: 25001879

9. Musso D, Nilles EJ, Cao-Lormeau VM. Rapid spread of emerging Zika virus in the Pacific area. Clinical microbiology and infection: the official publication of the European Society of Clinical Microbiology and Infectious Diseases. 2014;20(10):0595-6.

10. Oehler E, Watrin L, Larre P, Leparc-Goffart I, Lastere S, Valour F, et al. Zika virus infection complicated by GuillainBarre syndrome--case report, French Polynesia, December 2013. Euro surveillance: bulletin Europeen sur les maladies transmissibles. [European communicable disease bulletin]. 2014;19(9).

11. Dyer O. Zika virus spreads across Americas as concerns mount over birth defects.BMJ. 2015;351:h6983. DOI: 10.1136/bmj. h6983 PMID: 26698165

12. WHO. Zika virus outbreaks in the Americas.Wkly Epidemiol Rec. 2015;90(45):609-10.PMID: 26552108

13. World Health Organization (WHO). WHO Director-General summarizes the outcome of the Emergency Committee regarding clusters of microcephaly and Guillain-Barré syndrome; 1 Feb 2016. [Accessed 5 Feb 2016]. Available from: http://www.who.int/mediacentre/news/statements/2016/ emergency-committee-zika-microcephaly/en/

14. Renault P, Solet JL, Sissoko D, Balleydier E, Larrieu S, Filleul $\mathrm{L}$, et al. A major epidemic of chikungunya virus infection on Reunion Island, France, 2005-2006. Am J Trop Med Hyg. 2007;77(4):727-31.PMID: 17978079

15. Grard G, Caron M, Mombo IM, Nkoghe D, Mboui Ondo S, Jiolle D, et al. Zika virus in Gabon (Central Africa)--2007: a new threat from Aedes albopictus? PLoS Negl Trop Dis. 2014;8(2):e2681. DOI: 10.1371/journal.pntd.0002681 PMID: 24516683

16. Wong PS, Li MZ, Chong CS, Ng LC, Tan CH. Aedes (Stegomyia) albopictus (Skuse): a potential vector of Zika virus in Singapore.PLoS Negl Trop Dis. 2013;7(8):e2348. DOI: 10.1371/ journal.pntd.0002348 PMID: 23936579

17. Carrieri M, Albieri A, Angelini $P$, Baldacchini F, Venturelli C, Zeo SM, et al. Surveillance of the chikungunya vector Aedes albopictus (Skuse) in Emilia-Romagna (northern Italy): organizational and technical aspects of a large scale monitoring system. J Vector Ecol. 2011;36(1):108-16. DOI: 10.1111/j.1948-7134.2011.00147.x PMID: 21635648

18. Brottet E, Jaffar-Bandjee MC, Rachou E, Polycarpe D, Ristor B, Larrieu S, et al. Sentinel physician's network in Reunion Island: a tool for infectious diseases surveillance. Med Mal Infect. 2015;45(1-2):21-8. DOI: 10.1016/j.medmal.2014.11.004 PMID: 25575412

19. D’Ortenzio E, Renault P, Jaffar-Bandjee MC, Gauzere BA, Lagrange-Xelot $M$, Fouillet $A$, et al. A review of the dynamics and severity of the pandemic $A\left(\mathrm{H}_{1} \mathrm{~N}_{1}\right)$ influenza virus on Reunion island, 2009. Clinical microbiology and infection: the official publication of the European Society of Clinical Microbiology and Infectious Diseases. 2010;16(4):309-16.

20. Caillere N, Vilain P, Brottet E, Kaplon J, Ambert-Balay K, Polycarpe D, et al. A major outbreak of gastroenteritis in Reunion Island in 2012: first identification of $\mathrm{G} 12$ rotavirus on the Island. Euro surveillance: bulletin Europeen sur les maladies transmissibles. [European communicable disease bulletin]. 2013;18(19):20476.

21. Marguerite N, Brottet E, Pagès F, Jaffar-Bandjee MC, Schuffenecker I, Josset L, et al. A major outbreak of conjunctivitis caused by coxsackievirus A24, Réunion, January to April 2015. Euro Surveill. 2016;21(26):30271.PMID: 27387200

22. Charles-Smith LE, Reynolds TL, Cameron MA, Conway M Lau EH, Olsen JM, et al. Using Social Media for Actionable Disease Surveillance and Outbreak Management: A Systematic Literature Review. PLoS One. 2015;10(10):e0139701. DOI: 10.1371/journal.pone.0139701 PMID: 26437454

23. World Health Organization (WHO). International statistical classification of diseases and related health problems. 10th Revision. Volume 2. Instruction manual. 2010 ed. Geneva: WHO; 2011. Available from: http://www.who.int/ classifications/icd/ICD10Volume2_en_2010.pdf

24. Schuler-Faccini L, Ribeiro EM, Feitosa IM, Horovitz DD, Cavalcanti DP, Pessoa A, et al. , Brazilian Medical Genetics Society-Zika Embryopathy Task Force. Possible Association Between Zika Virus Infection and Microcephaly - Brazil, 2015. MMWR Morb Mortal Wkly Rep. 2016;65(3):59-62. DOl: 10.15585/mmwr.mm6503e2 PMID: 26820244 
25. Website Database EUROCAT. Data uploaded 6 Jan

2015). Available from: http://www.eurocat-network.eu/

ACCESSPREVALENCEDATA/PrevalenceTables (data uploaded 06/01/2015).

26. Bogoch II, Brady OJ, Kraemer MU, German M, Creatore MI,

Kulkarni MA, et al. Anticipating the international spread of Zika virus from Brazil. Lancet (London, England). 2016.

27. Hennessey M, Fischer M, Staples JE. Zika Virus Spreads to New Areas - Region of the Americas, May 2015-January 2016.MMWR Morb Mortal Wkly Rep. 2016;65(3):55-8. DOI: 10.15585/mmwr. mm6503e1 PMID: 26820163

\section{License and copyright}

This is an open-access article distributed under the terms of the Creative Commons Attribution (CC BY 4.0) Licence. You may share and adapt the material, but must give appropriate credit to the source, provide a link to the licence, and indicate if changes were made.

This article is copyright of the authors, 2016. 